\title{
The Design of Bullet Train Process Bogie and the Finite Element Analysis of Frame Strength
}

\author{
Jun DAI \\ Mechanical and Electrical Engineering Department, Tangshan College, Tang Shan, 0630000, \\ He Bei, China \\ 15081906410@163.com
}

Keyword: Process bogie, Frame, Strength, Finite element.

\begin{abstract}
The process bogie is a special equipment for bullet train maintenance. Frame is the main the main load-bearing frame in the process bogie, and its fatigue strength directly affects the safety performance of the entire process bogie. According to the actual maintenance needs, the project design a process bogie. Using the finite element analysis software ANSYS, the finite element model of frame is established, and the frame strength and rigidity is analyzed. Referring to the "Bullet train bogie frame strength test method" (TB/T2368-2005) and the Ministry of Railways standard "Railway vehicles strength design and test standard" (TB/T1335-1996), the frame strength is assessed. The analysis results are consistent with the practical application.
\end{abstract}

\section{Introduction}

With the rapid economic development of China, the development of high-speed railway has now entered a new stage. The safe and stable operation of bullet Train is becoming more and more important. Usually, the daily maintenance of the bullet train is implemented through putting the entire column into the overhaul base for simple maintenance and repair work, but the separation of the bullet train's body and bogie is needed when the maintenance level reaches three and above, so there is a need to develop a set of equipment which can carry traction body and enable the body to move between different locations.

The bullet train's process bogie is an special equipment which replaces the high-speed bogie of EMU and supports the train body to move between maintenance areas in the process state when different $\mathrm{CRH}$ electrical multiple units are in the process of decoding and overhaul, and different maintenance tasks of the high-speed bogie and the train body can be achieved as a result. Truss is one of the key components of process bogie, and it not only is the skeleton of the installation of various parts but also bears and passes the alternating vertical force, horizontal force and longitudinal force. The fatigue strength of truss directly affects the safety of the entire process bogie and is of great importance to the safety, reliability and economy of the railway locomotive vehicle maintenance. Therefore, according to the ministry of railway's standard-Power bogie frame strength test method TB/T2368-2005) and Railway vehicle strength design and test specification (TB/T1335-1996), static strength and fatigue strength of the process bogie's frame are analysed and the results can provide insight into the performance of the process bogie frame.

\section{The Overall Structure of Process Bogie}

Figure 1 shows the structure of the process bogie which is consisted of wheel frame, movable type II support, drive device, driving wheel set, driven wheel set, explosion-proof battery box, and electric control system and so on. The equipment bearing mesa is $180 \mathrm{~mm}$ height, $28 \mathrm{t}$ weight. The mesa width is adjustable, which meets the requirement of CRH1,2,3,5.

Work platform uses a fixed two vertical and one horizontal three beam structure with a support device fixed on the upper beam. The wheel axle box is fixed on the bottom of the longitudinal beam. Its structure is simple, bearing capacity is big, and have good matching installation with driving power and power plant. 


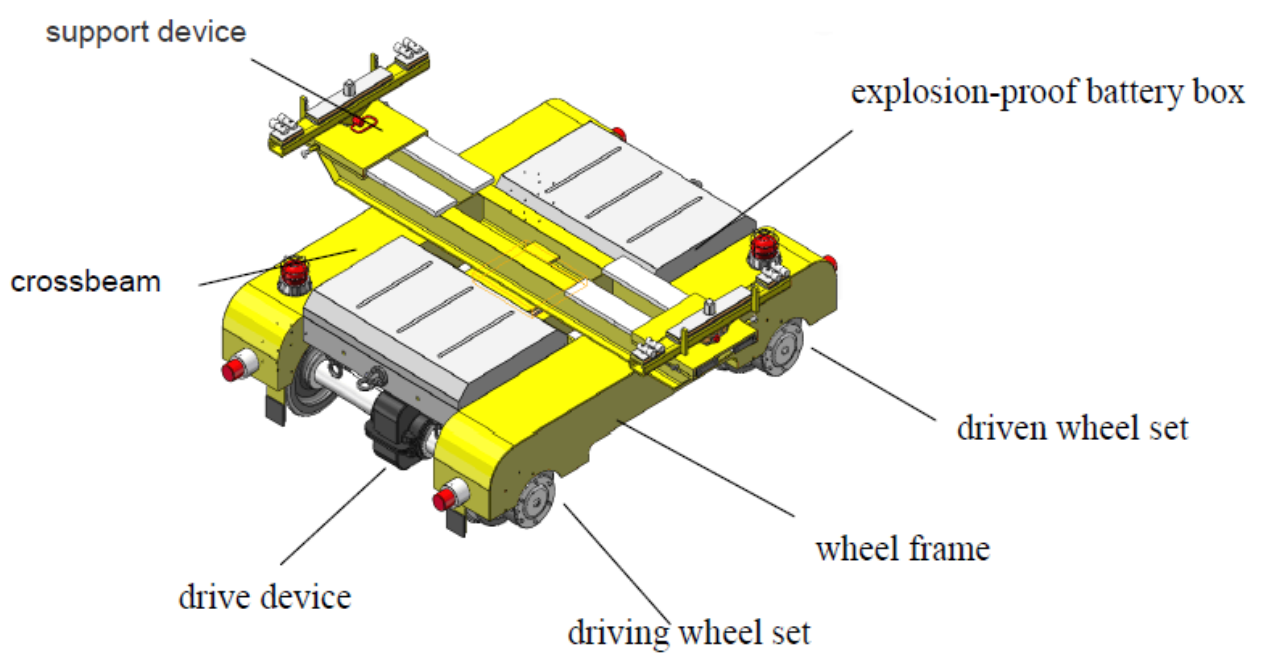

Fig.1 The overall structure of process bogie

\section{Framework Strength Analysis}

\section{The Construction of Finite Element Model of Framework}

Based on the structure character of the framework, it can be discretized into solid elements. The mesh of FEM is got by the ANSYS Workbench.SOLID187 element (10 nodes tetrahedral) is used. The number of the total discretized nodes of the framework is 553064, and the number of the elements is 275280 .

Considering the character that the truss is on the axle box rubber support, we set elastic boundary element on each of the supporting surface. The vertical, lateral and longitudinal stiffness of the boundary element are the three directional stiffness of a series of suspension. There are 12 elastic boundary elements[3]. Under different conditions, there is no restriction to the framework on other positions. In the model, $\mathrm{Z}$ coordinate is the forward direction of the vehicle, $\mathrm{Y}$ coordinate is the vertical upward direction, $\mathrm{X}$ coordinate is the transverse direction[2].

The solid model of the framework is shown in Figure2. The discretized FEM calculation and loading model is shown in Figure3.

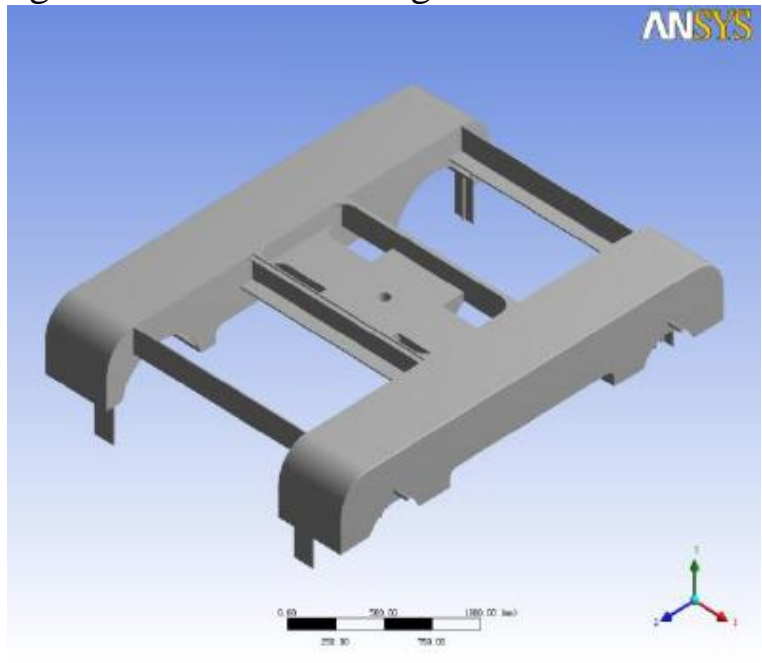

Fig. 2 The 3D model of the framework

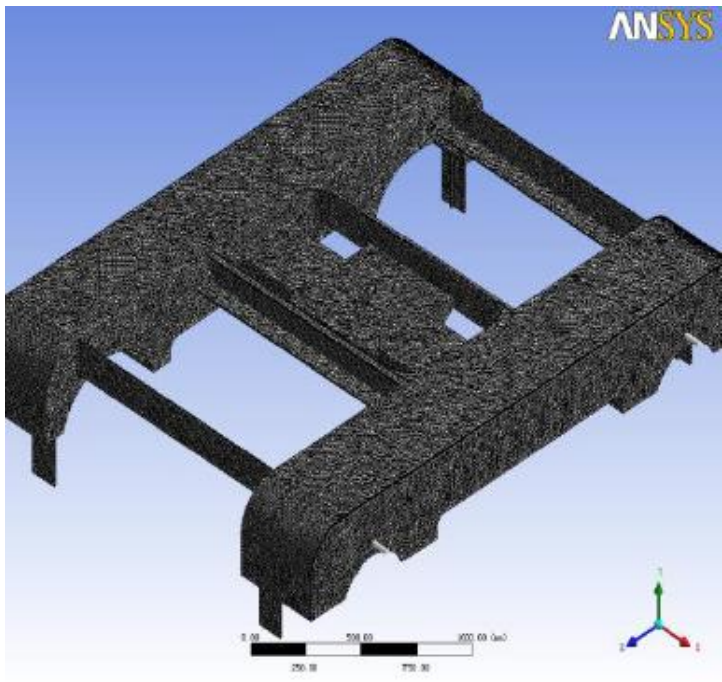

Fig. 3 The FEM model of the framework

\section{The FEM Calculated Load of the Framework}

According to the Power bogie frame's strength test method(TB/T2368-2005). When calculating the calculate the strength of the frame, we must calculate the vertical load, horizontal load in order 
to analyze the structure stress condition more comprehensive. The skew symmetric load is also considered in the calculation.

According to the design requirements, the vertical load borne by each frame is

$$
F=38.884 \times 9.81 / 2=190.73 \mathrm{KN}
$$

Vertical load acts, in the form of surface force, on installation surface of the frame's center plate.

The transverse load must be considered in the calculation of frame's strength, whose calculation formula is:

$$
F_{y}=0.5\left(F_{z}+0.5 m^{+} g\right)
$$

where $\mathrm{m}+$ is the weight of the frame itself which takes the value $5 \mathrm{t}$. Fz is the operating vertical load which takes $\mathrm{F} / 2=95.36 \mathrm{KN}$. As a result, each frame's operating transverse load calculated is $59.94 \mathrm{KN}$, and it acts on the center plate bearing surface.

Skew symmetric load is a set of counter-balanced vertical force which acts on the frame and is antisymmetric to the two axises of the frame. It takes the value $F_{x i e}=14.72 \mathrm{KN}$ in this calculation.

\section{The FEM's Design Condition of Frame}

According to the standard TB/T2368-2005, the rolling coefficient $\alpha$, in the normal operating condition, takes 0.10 , and the floating coefficient $\beta$ takes 0.20 . We only considered the floating of the train body, so the floating coefficient takes $\beta=0.20$. Table 1 shows six combined conditions adopted in the calculation of the condition of the frame's operating load.

Tab. 1 Main operating load's working condition 载荷组合表(单位:KN)

\begin{tabular}{|c|c|c|c|}
\hline $\begin{array}{c}\text { Working } \\
\text { condition }\end{array}$ & Vertical load & Transverse load & Skew symmetric load \\
\hline 1 & $F$ & & \\
\hline 2 & $(1+\beta) F$ & & \\
\hline 3 & $(1+\beta) F$ & $F_{y}$ & \\
\hline 4 & $(1+\beta) F$ & $-F_{y}$ & $F_{x i e}$ \\
\hline 5 & $(1+\beta) F$ & $F_{y}$ & $-F_{x i e}$ \\
\hline 6 & $(1+\beta) F$ & $-F_{y}$ & \\
\hline
\end{tabular}

\section{The Calculation of Fram'S Allowable Stress}

The allowable stress of material is the ratio of its yield limit $\sigma$ s to the safety coefficient $S$. Ordinary carbon steel (Q235) welding structure is usually adopted in the design of the frame, and its yield limit is $235 \mathrm{MPa}$.

According to the TB/T1335-1996, when calculating the complex mechanical components, the equivalent stress must be considered in calculation(Von Mises stress), and it must not exceed the allowable stress. All the stress results in this calculation are expressed by equivalent stress. The calculation formula for equivalent stress is:

$$
\sigma_{e}=\sqrt{0.5\left[\left(\sigma_{1}-\sigma_{2}\right)^{2}+\left(\sigma_{2}-\sigma_{3}\right)^{2}+\left(\sigma_{3}-\sigma_{1}\right)^{2}\right]}
$$

where, $\sigma \mathrm{e}$ is the equivalent stress, MPa; $\sigma \mathrm{i}$ is the principle stress $(\mathrm{i}=1,2,3), \mathrm{MPa}$. 
According to TB/T1335-1996, for the no weld area, the greatest possible load or the allowable stress under excessive load is the material's yield limit. Under the applied load, the allowable stress is the ratio of the material's yield limit to the 1.5 times of the safety factor. For the weld area, the greatest possible load or the allowable stress under excessive load is the ratio of the material's yield limit to the 1.1 times of the safety factor, and under the applied load, it is the ratio of the material's yield limit to the 1.65 times of the safety factor.

In this calculation, the working conditions are operating conditions, therefore under operating conditions, the allowable stress of the Q235 steel plate weld structure material is 142MPa.

The calculation result of the frame's stiffness

The structure of the frame will deform under the action of static load (operating condition 1), and the maximum deformation appeared on the center plate bearing surface of the frame $(4.247 \mathrm{~mm})$. The minimum deformation appeared in the position of frame's axle box $(1.935 \mathrm{~mm})$ shown in Figure3. In calculation the boundary element of the spring is set to linear stiffness, so it is very close to the stiff spring in practice. The relative displacement of the center bearing surface of the frame to the position of the axle box is $2.312 \mathrm{~mm}$. Seeing from the actual structure deformation and image of the deformation effect, the principle deformation is the vertical one, and the $2.312 \mathrm{~mm}$ relative displacement is ideal, so the stiffness of the frame is enough. Figure 4 and Figure 5 show the deformation cloud image of the frame in the first and second operating condition.

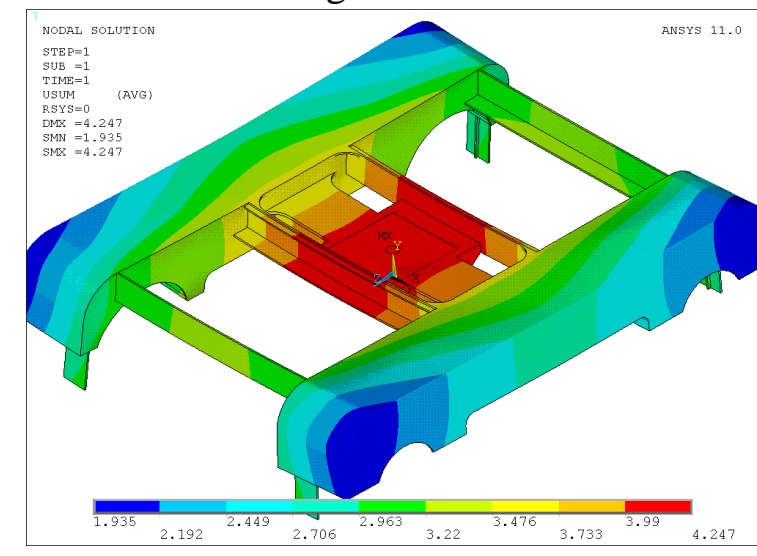

Fig. 4 Frame's deformation in the first operating condition

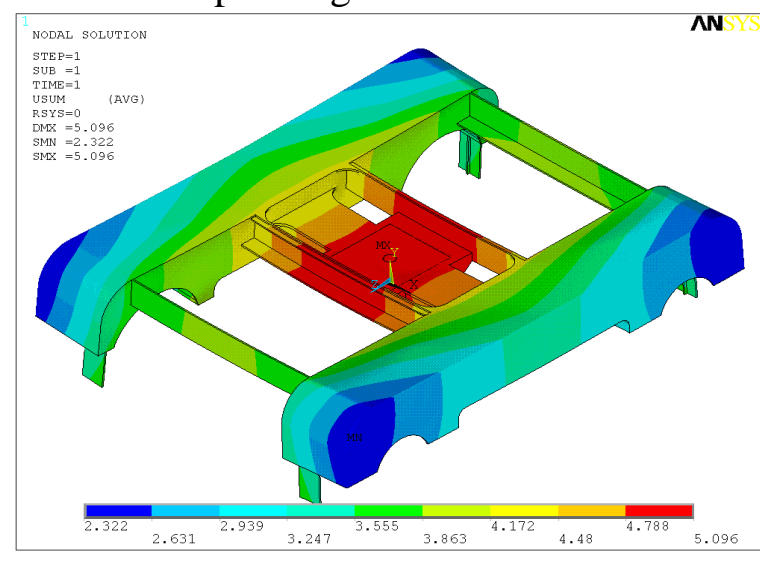

Fig. 5 Frame's deformation in the second operating condition

\section{The Calculation Result of the Frame'S Strength}

Figure 6 to Figure 11 and Table2 show the calculation result of the maximum equivalent stress of the frame's operating load condition. From the figure, we see that the maximum equivalent stress of the operating load condition is $116.719 \mathrm{MPa}$, and it appears in the joint between the frame's beam and side beam.

Tab. 2 The calculation results of the frame's maximum equivalent stress in operating load condition(MPa)

\begin{tabular}{|l|c|c|}
\hline $\begin{array}{c}\text { Calculation } \\
\text { conditions }\end{array}$ & $\begin{array}{c}\text { Maximum } \\
\text { equivalent } \\
\text { stress(MPa) }\end{array}$ & The maximum equivalent stress location \\
\hline Condition1 & 86.101 & The joint between the frame's beam and side beam \\
\hline Condition2 & 103.321 & The joint between the frame's beam and side beam \\
\hline Condition3 & 103.476 & The joint between the frame's beam and side beam \\
\hline Condition4 & 136.719 & The joint between the frame's beam and side beam \\
\hline Condition5 & 105.666 & The joint between the frame's beam and side beam \\
\hline Condition6 & 114.356 & The joint between the frame's beam and side beam \\
\hline
\end{tabular}


Condition1: $\mathrm{MAX}=86.101 \mathrm{MPa}$

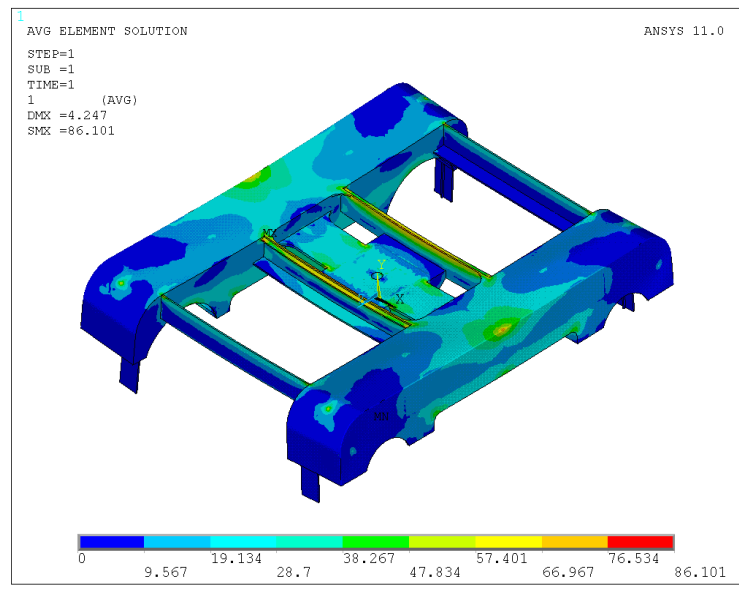

Fig. 6 von mises stress nephogram in condition1

Condition3: $\mathrm{MAX}=103.476 \mathrm{MPa}$

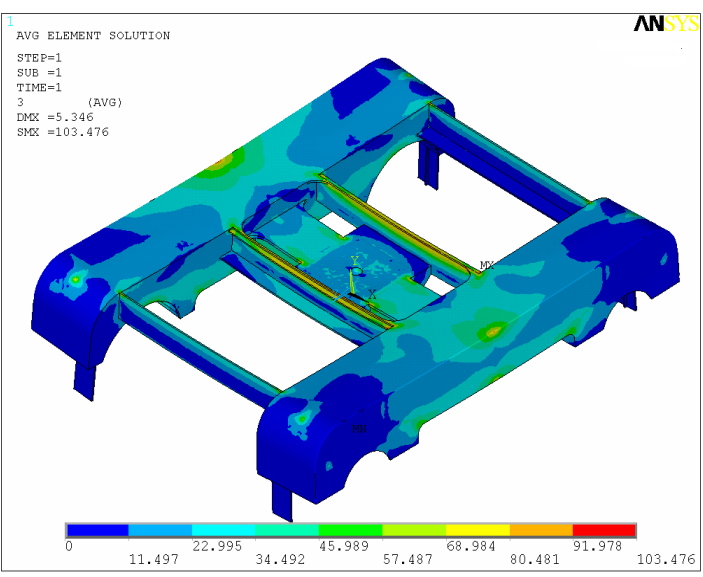

Fig. 8 von mises stress nephogram in condition 3

Condition5: $\mathrm{MAX}=105.666 \mathrm{MPa}$

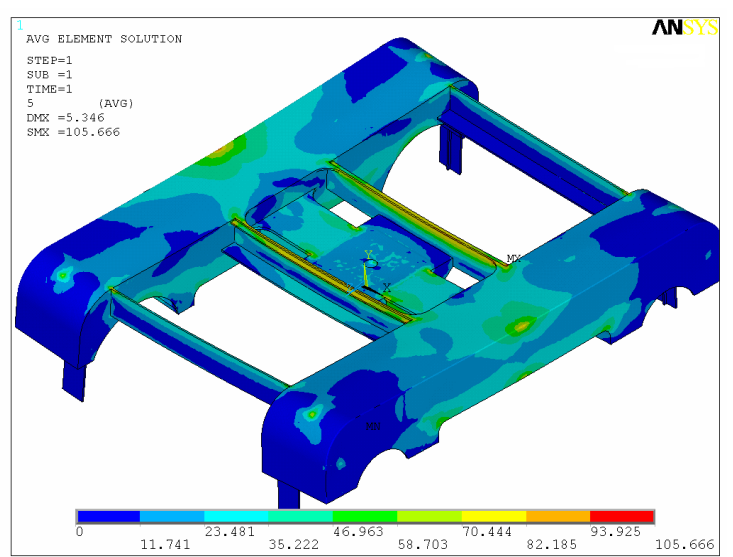

Fig. 10 von mises stress nephogram in condition5
Condition2: $\mathrm{MAX}=103.321 \mathrm{MPa}$

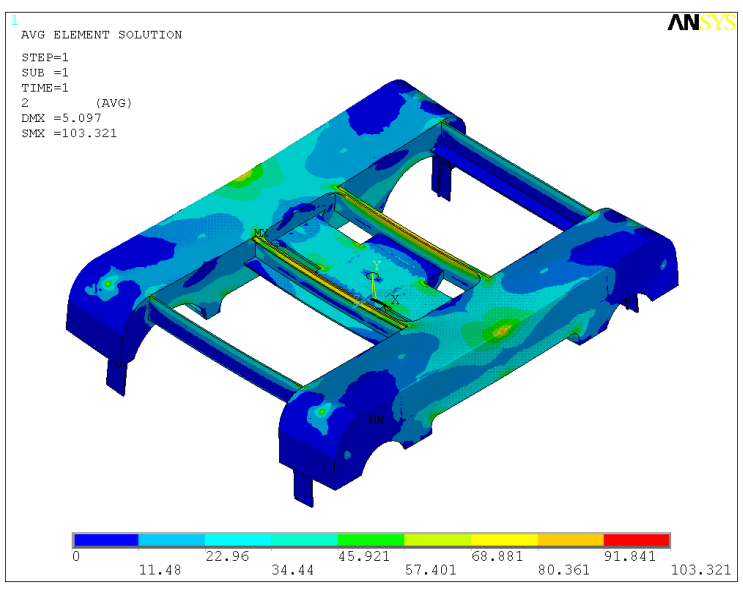

Fig. 7 von mises stress nephogram in condition 2

Condition4: $\mathrm{MAX}=136.719 \mathrm{MPa}$

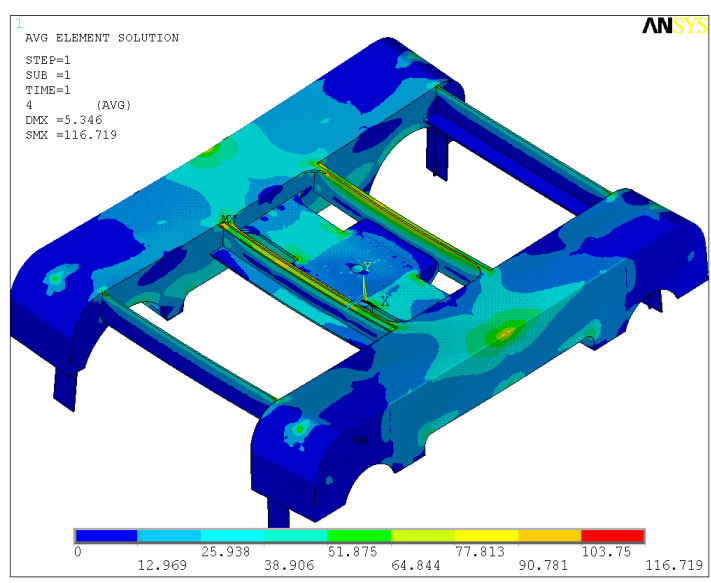

Fig. 9 von mises stress nephogram in condition 4

Condition6: $\mathrm{MAX}=114.356 \mathrm{MP}$

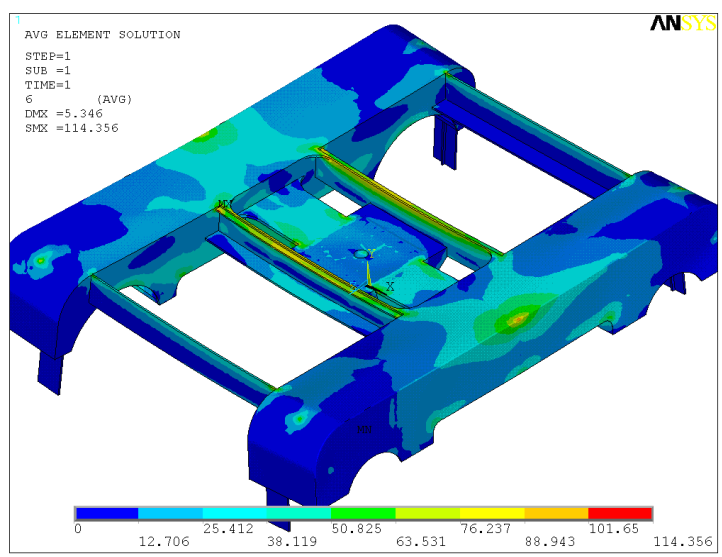

Fig. 11 von mises stress nephogram in condition6 


\section{Conclusion}

Through the frame strength analysis of the bullet train's process bogie frame, we can draw conclusions as follows:

When the frame is under static load, the maximum relative displacement is $2.312 \mathrm{~mm}$. From the stiffness calculation result of the frame's FEM model, the stiffness of the frame is enough.

In the working condition described in TB/T2368-2005, the maximum equivalent stress of the frame in working condition is $116.719 \mathrm{MPa}$, and it is lower than $142 \mathrm{MPa}$ which is the alloable stress standard of Q235 steel in working condition. The stiffness of the frame satisfied with the requirement.

\section{References}

[1] Xiang-hui song, wang hong,yue-jin shang. Train bogie frame strength analysis [J]. Mechanical Research \& Application, 2012, (01): 1-3.

[2] Shen yi. Application of adjustable process bogie joist [J]. Railway Locomotive workers [J], 2011(1): 4-6.

[3] Li tao. Since the technology oriented diameter bogie frame design [J]. Electric locomotive and the urban rail vehicles, 2003, (26): 25-27.

[4] Hua-jun luo, xi-hong chen, gong-an tao. The structure features and research strength of ZMA100 type bogie frame [J]. Electric locomotive and the urban rail vehicles, 2014, (01): 10-14.

[5] Qing-qun lan, wang wei, li rui. Track inspection car bogie frame strength experiment and fatigue assessment [J]. Modern Manufacturing Engineering, 2011 (02): 61-64.

[6] Hong-yan ye, ping-bo wu. Bogie weled frame static strength analysis and fatigue strength evaluation [J]. Railway Locomotive workers 2013, (10): 23-28.

[7] Zhong-ming lv, jing-quan bi. The railway passenger car bogie frame structure optimization analysis [J]. Railway vehicle, 2011, (07): 16-19.

[8] Railway industry standards of the People`s Republic of China, strength design and appraisal regulations for railway vehicle TB/T1335-1996. 